01.1;08.3;09.4

\title{
Анализ экситонной люминесценции в мезорезонаторах на основе GaN
}

\author{
(C) А.В. Белоновский ${ }^{1,2}$, К.М. Морозов ${ }^{2}$, Л.В. Котова ${ }^{2}$ \\ ${ }^{1}$ Санкт-Петербургский национальный исследовательский Академический университет им. Ж.И. Алфёрова РАН, \\ Санкт-Петербург, Россия \\ ${ }^{2}$ Университет ИТМО, Санкт-Петербург, Россия \\ E-mail: leha.s92.92@gmail.com
}

Поступило в Редакцию 23 сентября 2021 г.

В окончательной редакции 8 октября 2021 r.

Принято к публикации 8 октября 2021 г.

Рассмотрено взаимодействие экситонной моды с собственными фотонными модами в структуре на основе GaN с размерами в несколько микрометров. Продемонстрирована методика регистрации спектров излучения таких структур. Подобраны наиболее оптимальные форма и размеры резонатора для эффективного взаимодействия света и вещества. Получен и проанализирован теоретический спектр для подобранного резонатора.

Ключевые слова: нитрид галлия, экситон, мезорезонатор, спектр.

DOI: 10.21883/PJTF.2022.01.51881.19033

Исследования в области взаимодействия света с веществом имеют сегодня важное фундаментальное и прикладное значение в физике. Одним из основных элементов, в котором может происходить эффективное взаимодействие света и вещества, являются оптические микрорезонаторы. Такие структуры имеют размеры порядка длины волны излучения в материале. Встраивание эмиттера внутрь микрорезонатора может привести к различного рода эффектам, связанным с взаимодействием света и вещества. За счет увеличения плотности фотонных состояний в области расположения эмиттера в микрорезонаторе по сравнению с плотностью фотонных состояний в вакууме увеличивается скорость спонтанного излучения. Увеличение или уменьшение скорости спонтанного излучения в резонаторе называется эффектом Парселла [1]. В 1992 г. Вайсбушем и др. наблюдался эффект сильной связи между экситонами в квантовой яме и собственными фотонными модами планарного микрорезонатора [2]. Обширные исследования в этом направлении в течение последних трех десятилетий сформировали новую парадигму в физике твердого тела - квантовую электродинамику кристаллов [3-5]. В микрорезонаторах электромагнитные поля локализованы в объеме, сравнимом с длиной волны света. Сильная связь в микрорезонаторе реализуется между экситоном в квантовой яме и только одной модой резонатора [2], в более сложных конструкциях (например, в связанных резонаторах) [6] возможно взаимодействие между несколькими экситонами и модами резонатора. Расщепление Раби будет меньше, чем расстояние между модами резонатора. Для достижения этих условий требуется изготавливать структуры малых размеров (порядка нескольких микрометров). Однако изготовление структур таких размеров на данный момент является сложной технологической задачей для многих типов полупроводников [7], например для широкозонных полупроводников $\mathrm{GaN}$, которые обладают такими полезными свойствами, как яркое излучение в УФ-диапазоне, большая сила осциллятора экситонов, химическая и термическая стабильность. Между тем малоизученными остаются резонаторы с размерами в несколько длин волн, где экситонная мода взаимодействует сразу с несколькими оптическими модами системы (мезорезонаторы).

В первых исследованиях мезорезонаторов [8-10] был показан ряд новых эффектов: нелинейное поведение населенностей мод системы; особый, немонотонный характер бистабильного поведения поляритонной населенности в зависимости от накачки; возможность реализации сильной и слабой связи между экситонной модой и собственными фотонными модами системы, несмотря на высокую плотность мод резонатора.

Гамильтониан для системы, описывающей взаимодействие экситонной моды с энергией $\hbar \omega_{k}$ и нескольких фотонных мод с энергиями $\hbar \omega_{k}$, выглядит следующим образом [11]:

$$
\hat{H}=\hbar \omega_{x} \hat{x}^{+} \hat{x}+\sum_{k} \hbar \omega_{k} \hat{a}_{k}^{+} \hat{a}_{k}+\sum_{k} \hbar g_{k}\left(\hat{a}_{k}^{+} \hat{x}+\hat{a}_{k} \hat{x}^{+}\right),
$$

где $\hat{x}^{+}, \hat{a}_{k}^{+}\left(\hat{x}, \hat{a}_{k}\right)$ - операторы рождения (уничтожения) экситона и фотона соответственно. Для данных операторов справедливы следующие коммутационные соотношения: $\left[\hat{a}_{k}, \hat{a}_{k}^{+}\right]=1,\left[\hat{x}, \hat{x}^{+}\right]=1, \hbar g_{k}-$ энергия взаимодействия экситонной моды и $k$-й фотонной моды. При наличии диссипации система модернизируется до описания системы с помощью лиувиллиана для матрицы плотности с членами, отвечающими за диссипацию. Следовательно, полное состояние системы описывается 
матрицей плотности $\hat{\rho}$, а ее эволюция определяется уравнением $\partial_{t} \hat{\rho}=\hat{\mathscr{L}} \hat{\rho}$, где $\hat{\mathscr{L}}$ - лиувиллиан с членами Линдблада, описывающими диссипацию:

$$
\begin{aligned}
\hat{\mathscr{L}} \hat{\rho} & =\frac{i}{\hbar}[\hat{\rho}, \hat{H}]+\frac{\gamma_{x}}{2}\left(2 \hat{x} \hat{\rho} \hat{x}^{+}-\hat{x}^{+} \hat{x} \hat{\rho}-\hat{\rho} \hat{x}^{+} \hat{x}\right) \\
& +\sum_{k} \frac{\gamma_{k}}{2}\left(2 \hat{a}_{k} \hat{\rho} \hat{a}_{k}^{+}-\hat{a}_{k}^{+} \hat{a}_{k} \hat{\rho}-\hat{\rho} \hat{a}_{k}^{+} \hat{a}_{k}\right),
\end{aligned}
$$

где $\gamma_{x}, \gamma_{k}-$ коэффициенты диссипации для экситонной и фотонной мод соответственно.

Для нахождения среднего значения числа частиц в экситонной и фотонной модах, а также в их смешанных состояниях необходимо решить систему дифференциальных уравнений, которая получается из соотношения $\partial_{t}\langle\hat{O}\rangle=\operatorname{Tr}\left(\hat{O} \partial_{t} \hat{\rho}\right)=\operatorname{Tr}(\hat{O} \hat{\mathscr{L}} \hat{\rho})$, где $\hat{O}-$ произвольный оператор. Система уравнений, описывающая временну́ю динамику населенности мод, тогда будет выглядеть следующим образом:

$$
\left\{\begin{array}{l}
\partial_{t} n_{x x}=\sum_{k} i g_{k}\left(n_{k x}-n_{x k}\right)-\gamma_{x} n_{x x}, \\
\partial_{t} n_{i j}=i\left(\omega_{i}-\omega_{j}\right) n_{i j}-i g_{j} n_{i x}+i g_{i} n_{x j}-\frac{1}{2}\left(\gamma_{i}+\gamma_{j}\right) n_{i j}, \\
\partial_{t} n_{x i}=i\left(\omega_{x}-\omega_{i}\right) n_{x i}+\sum_{k} i g_{k} n_{k i}-i g_{i} n_{x x}-\frac{1}{2}\left(\gamma_{x}+\gamma_{i}\right) n_{x i},
\end{array}\right.
$$

где $n_{x x}=\left\langle\hat{x}^{+} \hat{x}\right\rangle-$ среднее значение числа экситонов, $n_{x i}=\left\langle\hat{x}^{+} \hat{a}_{i}\right\rangle=n_{i x}^{*}$ и $n_{i j}=\left\langle\hat{a}_{i}^{+} \hat{a}_{j}\right\rangle(i \neq j)$ - значения в смешанных состояниях. Количество фотонов в моде $i$ описывается выражением $n_{i i}=\left\langle\hat{a}_{i}^{+} \hat{a}_{i}\right\rangle$

Для расчета спектров излучения мезорезонатора использовался формализм, описанный в [12]. Спектр излучения системы (плотность вероятности того, что излучаемый системой фотон имеет частоту $\omega$ ) с учетом нормировки будет выглядеть следующим образом:

$$
S(\omega)=\frac{\sum_{i} s_{i}(\omega)}{\sum_{i} \int_{0}^{\infty} n_{i i} d t}
$$

Значение функции $s_{i}(\omega)$ можно получить с помощью корреляционной функции первого порядка. Подробный расчет корреляционных функций для функции $s_{i}(\omega)$ приведен в работе [12]. Таким образом, с помощью описанной выше методики можно получать спектры излучения для систем, в которых происходит взаимодействие одной экситонной и нескольких фотонных мод.

Большинство работ по выращиванию планарных структур из $\mathrm{GaN}$ показало, что выращенные резонаторы будут иметь шестиугольную форму ввиду особенности кристаллической решетки $[13,14]$. Благодаря методике селективной газофазной эпитаксии из металлоорганических соединений в экспериментах удается получать шестиугольные полоски разной длины. Однако уменьшение симметрии формы резонатора приводит к резкому уменьшению добротности собственных мод. В настоящей работе будем исследовать гексагональные симметричные структуры из $\mathrm{GaN}$, которые, как показывает
Энергия $\hbar \omega$ и добротность $Q$ высокодобротной моды в зависимости от длины стороны гексагонального мезорезонатора $L$

\begin{tabular}{c|c|c}
\hline$L, \mu \mathrm{m}$ & $\hbar \omega, \mathrm{eV}$ & $Q$ \\
\hline 1.5 & 3.5099 & 11501 \\
2.0 & 3.4969 & 1791 \\
2.5 & 3.4833 & 50068 \\
3.0 & 3.4727 & $1.13 \cdot 10^{6}$ \\
3.5 & 3.4519 & $4.5 \cdot 10^{5}$
\end{tabular}

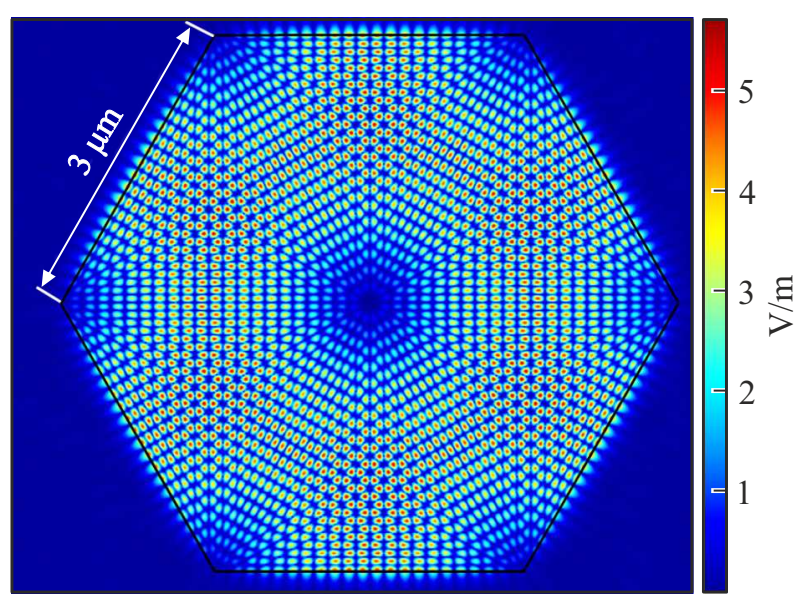

Рис. 1. Распределение интенсивности электромагнитного поля внутри идеального гексагонального резонатора $\mathrm{GaN}$ со стороной $3 \mu \mathrm{m}$ и показателем преломления $n=2.6267$, рассчитанное с помощью COMSOL. Энергия $3.4727 \mathrm{eV}, Q=1.13 \cdot 10^{6}$.

эксперимент, вырастить гораздо проще, чем структуры другой геометрии. Также в гексагональных структурах гораздо вероятнее появление одной высокодобротной моды, которая может эффективно взаимодействовать с экситоном [8].

В первую очередь определим размеры гексагональной структуры, в которой реализуется такая структура мод, где высокодобротная мода будет иметь энергию, близкую к энергии экситона. Расчет собственных мод и их добротностей для двумерного гексагонального мезорезонатора проводился с помощью моделирования в COMSOL Multiphysics. Показатель преломления для мезорезонатора $n_{\text {cavity }}=2.6267$, поглощение отсутствует. Окружающая среда имеет показатель преломления $n_{\text {media }}=1$. В таблице показаны рассчитанные значения энергии $\hbar \omega$ и добротности $Q$ высокодобротной моды резонатора для нескольких длин стороны гексагонального мезорезонатора $L$. Как видно из таблицы, для размера $L=3 \mu \mathrm{m}$ энергия высокодобротной моды $\hbar \omega=3.4727 \mathrm{eV}$ наиболее близка к энергии экситонной моды в $\mathrm{GaN} \hbar \omega_{x}=3.47 \mathrm{eV}$ [13]. На рис. 1 показано распределение интенсивности электромагнитного поля внутри резонатора для данной высокодобротной моды с $Q=1.13 \cdot 10^{6}$. Также из таблицы видно, что при увеличении мезорезонатора значительно увеличивается 


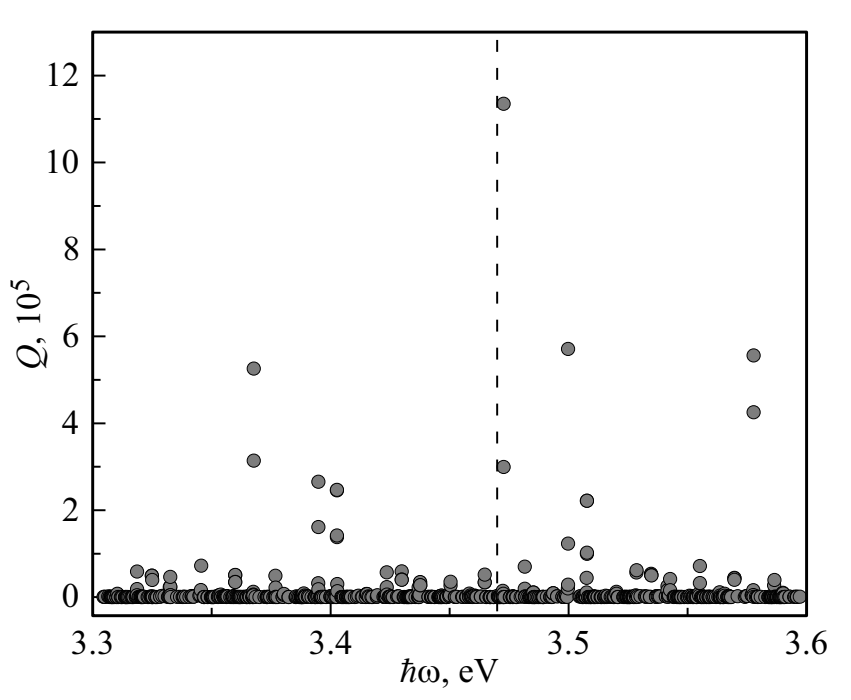

Рис. 2. Распределение собственных мод гексагонального мезорезонатора по энергиям и добротностям. Штриховой линией показана энергия экситонной моды $\hbar \omega_{x}=3.47 \mathrm{eV}$.

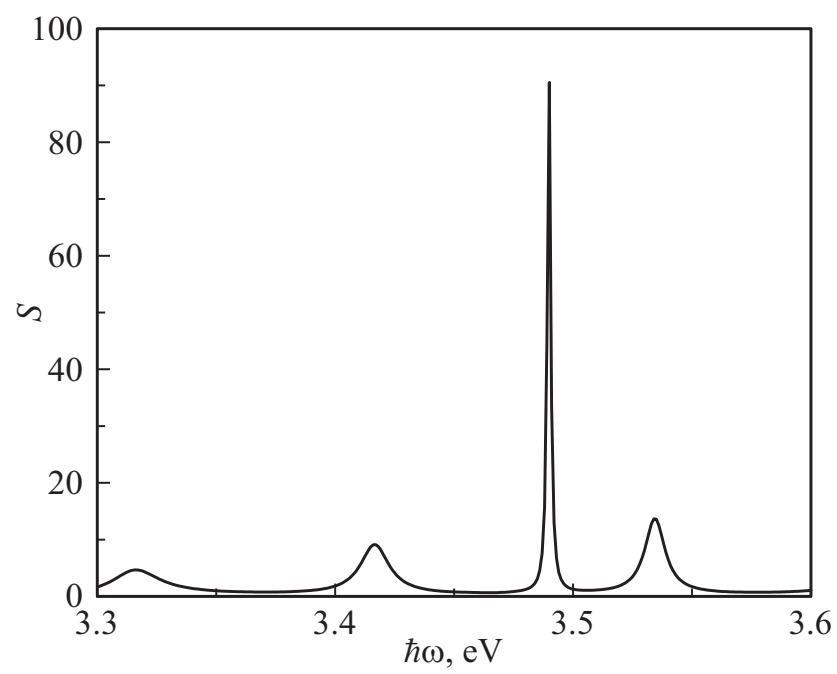

Рис. 3. Теоретически рассчитанный спектр излучения от подобранного гексагонального мезорезонатора.

добротность мод. Однако при этом будет уменьшаться энергетический интервал между модами, в том числе высокодобротными. Поэтому для наиболее оптимального мезорезонатора $L=3 \mu \mathrm{m}$ было рассчитано распределение собственных оптических мод по энергиям и добротностям (рис. 2). Как видно из рис. 2 , существует всего одна высокодобротная мода в области энергий экситонной моды. Тем не менее существует несколько собственных мод с добротностью, всего в 2 раза меньшей, чем добротность основной моды.

Для расчета спектра найденного мезорезонатора были взяты следующие значения параметров: энергия экситонной моды $\hbar \omega_{x}=3.47 \mathrm{eV}$, коэффициент диссипации для экситона $\gamma_{x}=0.075 \mathrm{meV}$ [11], константа связи $\hbar g_{k} \approx 50 \mathrm{meV}$ [15]. Коэффициенты диссипации для фотонных мод $\gamma_{k}=\frac{\omega_{k}}{Q_{k}}$ (температура равна нулю). Начальные значения числа экситонов в системе $n_{x x}^{0}=10$ и фотонов $n_{i i}^{0}=0$. Таким образом, мы рассматриваем систему, в которую изначально было инжектировано некоторое количество экситонов. Поскольку мы рассматриваем линейную модель, количество начальных экситонов не влияет на динамику населенностей фотонных и экситонных мод. На рис. 3 показан рассчитанный спектр излучения подобранного мезорезонатора. Для того чтобы определить, каким образом произошло расщепление по энергии между высокодобротной фотонной модой и экситонной модой, производились манипуляции с величиной константы связи. В результате видно, что поляритонным модам соответствуют энергии $\hbar \omega_{p 1}=3.49 \mathrm{eV}$ и $\hbar \omega_{p 2}=3.53 \mathrm{eV}$. Увеличение интенсивности излучения для моды $\hbar \omega_{p 1}$ по сравнению с интенсивностью излучения для моды $\hbar \omega_{p 2}$ и сдвиг мод по энергии связаны с наличием в структуре других мод с меньшей добротностью. Результаты моделирования в двух измерениях могут быть применимы для плоских резонаторов, в которых на конфигурацию мод системы влияет в основном только форма резонатора по двум измерениям [16].

Таким образом, в работе подобрана оптимальная форма мезорезонатора на основе $\mathrm{GaN}$ с учетом особенностей получения данных структур в экспериментах. Определен размер мезорезонатора из $\mathrm{GaN}$, в котором образуется наиболее подходящая структура собственных мод системы. В рассчитанной структуре собственных фотонных мод в области энергии экситонной моды существует одна высокодобротная оптическая мода, которая может эффективно взаимодействовать с экситоном. В полученном спектре наблюдается усиление интенсивности излучения для поляритонной моды с энергией $\hbar \omega_{p 1}=3.49 \mathrm{eV}$.

\section{Финансирование работы}

Работа поддержана Министерством науки и высшего образования РФ (проект 0791-2020-0008).

\section{Конфликт интересов}

Авторы заявляют, что у них нет конфликта интересов.

\section{Список литературы}

[1] E.M. Purcell, Phys. Rev., 69, 681 (1946).

[2] C. Weisbuch, M. Nishioka, A. Ishikava, Y. Arakawa, Phys. Rev. Lett., 69 (23), 3314 (1992). DOI: 10.1103/PhysRevLett.69.3314

[3] J. Kasprzak, M. Richard, S. Kundermann, A. Baas, P. Jeambrun, J.M.J. Keeling, F.M. Marchetti, M.H. Szymańska, R. André, J.L. Staehli, V. Savona, P.B. Littlewood, B. Deveaud, L.S. Dang, Nature, 443 (7110), 409 (2006).

DOI: $10.1038 /$ nature 05131 
[4] A. Amo, J. Lefrére, S. Pigeon, C. Adrados, C. Ciuti, I. Carusotto, R. Houdré, E. Giaobino, A. Bramati, Nature Phys., 5 (11), 805 (2009). DOI: 10.1038/nphys 1364

[5] M. Sich, D.N. Krizhanovskii, M.S. Skolnick, A.V. Gorbach, R. Hartley, D.V. Skryabin, E.A. Cerda-Méndez, K. Biermann, R. Hey, P.V. Santos, Nature Photon., 6 (1), 50 (2011). DOI: $10.1038 /$ nphoton.2011.267

[6] A. Armitage, M.S. Skolnick, V.N. Astratov, D.M. Whittaker, G. Panzarini, L.C. Andreani, T.A. Fisher, J.S. Roberts, A.V. Kavokin, M.A. Kaliteevski, M.R. Vladimirova, Phys. Rev. B, 57 (23), 14877 (1998). DOI: 10.1103/PhysRevB.57.14877

[7] T. Takeuchi, S. Kamiyama, M. Iwaya, I. Akasaki, Rep. Prog. Phys., 82 (1), 012502 (2019). DOI: $10.1088 / 1361-6633 /$ aad3e9

[8] A.V. Belonovski, I.V. Levitskii, K.M. Morozov, G. Pozina, M.A. Kaliteevski, Opt. Express, 28 (9), 12688 (2020). DOI: $10.1364 /$ OE.388899

[9] G. Pozina, C. Hemmingsson, A.V. Belonovskii, I.V. Levitskii, M.I. Mitrofanov, E.I. Girshova, K.A. Ivanov, S.N. Rodin, K.M. Morozov, V.P. Evtikhiev, M.A. Kaliteevski, Phys. Status Solidi A, 217 (14), 1900894 (2019).

DOI: $10.1002 /$ pssa.201900894

[10] A.V. Belonovski, K.M. Morozov, E.I. Girshova, G. Pozina, M.A. Kaliteevski, Opt. Express, 29 (13), 20724 (2021). DOI: $10.1364 /$ OE.420277

[11] A. Baas, J.P. Karr, H. Eleuch, E. Giacobino, Phys. Rev. A, 69 (2), 023809 (2004). DOI: 10.1103/PhysRevA.69.023809

[12] F.P. Laussy, E. del Valle, C. Tejedor, Phys. Rev. B, 79 (23), 235325 (2009). DOI: 10.1103/PhysRevB.79.235325

[13] G. Pozina, K.A. Ivanov, M.I. Mitrofanov, M.A. Kaliteevski, K.M. Morozov, I.V. Levitskii, G.V. Voznyuk, V.P. Evtikhiev, S.N. Rodin, Phys. Status Solidi B, 256 (6), 1800631 (2019). DOI: $10.1002 /$ pssb.201800631

[14] В.В. Лундин, А.Ф. Цацульников, С.Н. Родин, А.В. Сахаров, С.О. Усов, М.И. Митрофанов, Я.В. Левицкий, В.П. Евтихиев, ФТП, 52 (10), 1237 (2018). DOI: $10.21883 /$ FTP.2018.10.46467.8861 [W.V. Lundin, A.F. Tsatsulnikov, S.N. Rodin, A.V. Sakharov, S.O. Usov, M.I. Mitrofanov, I.V. Levitskii, V.P. Evtikhiev, Semiconductors, 52 (10), 1357 (2018). DOI: 10.1134/S106378261810007X].

[15] M.A. Kaliteevski, K.A. Ivanov, G. Pozina, A.J. Gallant, Sci Rep., 4, 5444 (2014). DOI: 10.1038/srep05444

[16] А.В. Белоновский, Г. Позина, Я.В. Левитский, К.М. Морозов, М.И. Митрофанов, Е.И. Гиршова, К.А. Иванов, С.Н. Родин, В.П. Евтихиев, М.А. Калитеевский, ФТП, 54 (1), 85 (2020). DOI: 10.21883/FTP.2020.01.48780.9257 [A.V. Belonovskii, G. Pozina, I.V. Levitskii, K.M. Morozov, M.I. Mitrofanov, E.I. Girshova, K.A. Ivanov, S.N. Rodin, V.P. Evtikhiev, M.A. Kaliteevski, Semiconductors, 54 (1), 127 (2020). DOI: 10.1134/S1063782620010042]. 